\title{
Generalization of Two Types of Improper Integrals
}

\author{
Chii-Huei Yu ${ }^{1, *}$, Bing-Huei Chen ${ }^{2}$ \\ ${ }^{1}$ Department of Management and Information, Nan Jeon University of Science and Technology, Tainan City, 73746, Taiwan \\ ${ }^{2}$ Department of Electrical Engineering, Nan Jeon University of Science and Technology, Tainan City, 73746, Taiwan \\ *Corresponding Author: chiihuei@mail.nju.edu.tw
}

Copyright (C) 2014 Horizon Research Publishing All rights reserved.

\begin{abstract}
This article uses the mathematical software Maple for the auxiliary tool to study two types of improper integrals. We can obtain the infinite series forms of these two types of integrals by using geometric series, differentiation term by term, and differentiation with respect to a parameter. On the other hand, we provide some examples to do calculation practically. The research methods adopted in this study involved finding solutions through manual calculations and verifying these solutions by using Maple. This type of research method not only allows the discovery of calculation errors, but also helps modify the original directions of thinking from manual and Maple calculations. For this reason, Maple provides insights and guidance regarding problem-solving methods.
\end{abstract}

Keywords Improper Integrals, Infinite Series Forms, Geometric Series, Differentiation Term by Term, Differentiation with Respect to a Parameter, Maple

\section{Introduction}

The computer algebra system (CAS) has been widely employed in mathematical and scientific studies. The rapid computations and the visually appealing graphical interface of the program render creative research possible. Maple possesses significance among mathematical calculation systems and can be considered a leading tool in the CAS field. The superiority of Maple lies in its simple instructions and ease of use, which enable beginners to learn the operating techniques in a short period. In addition, through the numerical and symbolic computations performed by Maple, the logic of thinking can be converted into a series of instructions. The computation results of Maple can be used to modify our previous thinking directions, thereby forming direct and constructive feedback that can aid in improving understanding of problems and cultivating research interests. Inquiring through an online support system provided by Maple or browsing the Maple website (www.maplesoft.com) can facilitate further understanding of Maple and might provide unexpected insights. As for the instructions and operations of Maple, we can refer to [1-7].
In [8], there are the following formulas of two types of improper integrals

$$
\begin{aligned}
& \int_{0}^{\infty} \frac{\sin a x}{\sinh b x} d x=\frac{\pi}{2 b} \tanh \frac{a \pi}{2 b} \\
& \int_{0}^{\infty} \frac{\cos a x}{\cosh b x} d x=\frac{\pi}{2 b} \operatorname{sech} \frac{a \pi}{2 b}
\end{aligned}
$$

where $a, b$ are real numbers, and $a \neq 0, b>0$. In this study, we generalize the above improper integrals (1) and (2) to the following two types of improper integrals

$$
\begin{aligned}
& \int_{0}^{\infty} \frac{x^{n} \sin \left(a x+\frac{n \pi}{2}\right)}{\sinh b x} d x \\
& \int_{0}^{\infty} \frac{x^{n} \cos \left(a x+\frac{n \pi}{2}\right)}{\cosh b x} d x
\end{aligned}
$$

where $a, b$ are real numbers, $a \neq 0, b>0$, and $n$ is any non-negative integer. We can obtain the infinite series forms of these two types of improper integrals by using geometric series, differentiation term by term, and differentiation with respect to a parameter; these are the major results of this study (i.e., Theorems 1 and 2). The study of related integral problems can refer to [9-29]. On the other hand, we propose four examples to do calculation practically. The research methods adopted in this study involved finding solutions through manual calculations and verifying these solutions by using Maple. This type of research method not only allows the discovery of calculation errors, but also helps modify the original directions of thinking from manual and Maple calculations. Therefore, Maple provides insights and guidance regarding problem-solving methods.

\section{Main Results}

Firstly, we introduce three important theorems used in this study.

\subsection{Geometric Series}




$$
\frac{1}{1+u}=\sum_{k=0}^{\infty}(-1)^{k} u^{k}
$$

where $u$ is a real number, and $|u|<1$.

\subsection{Differentiation Term by Term ([30])}

For all non-negative integers $k$, if the functions $g_{k}:(a, b) \rightarrow R$ satisfy the following three conditions : (i) there exists a point $x_{0} \in(a, b)$ such that $\sum_{k=0}^{\infty} g_{k}\left(x_{0}\right)$ is convergent, (ii) all functions $g_{k}(x)$ are differentiable on open interval $(a, b)$, (iii) $\sum_{k=0}^{\infty} \frac{d}{d x} g_{k}(x)$ is uniformly convergent on $(a, b)$. Then $\sum_{k=0}^{\infty} g_{k}(x)$ is uniformly convergent and differentiable on $(a, b)$. Moreover, its derivative $\frac{d}{d x} \sum_{k=0}^{\infty} g_{k}(x)=\sum_{k=0}^{\infty} \frac{d}{d x} g_{k}(x)$.

\subsection{Differentiation with Respect to A Parameter ([31])}

Suppose $c, d, \lambda, \beta$ are real numbers and the function $f(a, x)$ is defined on $[c, d] \times[\lambda, \beta]$. If $f(a, x)$ and its partial derivative $\frac{\partial f}{\partial a}(a, x)$ are continuous functions on $[c, d] \times[\lambda, \beta]$. Then $F(a)=\int_{\lambda}^{\beta} f(a, x) d x$ is differentiable on the open interval $(c, d)$, and its derivative $\frac{d}{d a} F(a)=$ $\int_{\lambda}^{\beta} \frac{\partial f}{\partial a}(a, x) d x$ for all $a \in(c, d)$.

The following is the first result in this study, we determine the infinite series form of the improper integral (3).

\subsection{Theorem 1}

Suppose $a, b$ are real numbers, $a \neq 0, b>0$, and $n$ is any non-negative integer.

Case (A): If $a>0$. Then the improper integral

$$
\begin{gathered}
\int_{0}^{\infty} \frac{x^{n} \sin \left(a x+\frac{n \pi}{2}\right)}{\sinh b x} d x \\
=\frac{(-1)^{n} \pi^{n+1}}{b^{n+1}} \cdot \sum_{k=0}^{\infty}(-1)^{k} k^{n} \exp \left(-\frac{a k \pi}{b}\right)
\end{gathered}
$$

Case (B): If $a<0$. Then

$$
\int_{0}^{\infty} \frac{x^{n} \sin \left(a x+\frac{n \pi}{2}\right)}{\sinh b x} d x
$$

$$
=-\frac{\pi^{n+1}}{b^{n+1}} \cdot \sum_{k=0}^{\infty}(-1)^{k} k^{n} \exp \left(\frac{a k \pi}{b}\right)
$$

2.4.1. Proof

Case (A): If $a>0$. Because

$$
\begin{gathered}
\int_{0}^{\infty} \frac{\sin a x}{\sinh b x} d x \\
=\frac{\pi}{2 b} \tanh \frac{a \pi}{2 b}(\operatorname{By}(1)) \\
=\frac{\pi}{2 b} \cdot \frac{\exp \left(\frac{a \pi}{2 b}\right)-\exp \left(-\frac{a \pi}{2 b}\right)}{\exp \left(\frac{a \pi}{2 b}\right)+\exp \left(-\frac{a \pi}{2 b}\right)} \\
=\frac{\pi}{2 b} \cdot \frac{1-\exp \left(-\frac{a \pi}{b}\right)}{1+\exp \left(-\frac{a \pi}{b}\right)} \\
=\frac{\pi}{2 b} \cdot\left[-1+\frac{2}{1+\exp \left(-\frac{a \pi}{b}\right)}\right] \\
\frac{\pi}{2 b} \cdot\left[-1+2 \cdot \sum_{k=0}^{\infty}(-1)^{k} \exp \left(-\frac{a k \pi}{b}\right)\right]
\end{gathered}
$$

(Using geometric series) (7)

$$
=-\frac{\pi}{2 b}+\frac{\pi}{b} \cdot \sum_{k=0}^{\infty}(-1)^{k} \exp \left(-\frac{a k \pi}{b}\right)
$$

By differentiation term by term and differentiation with respect to a parameter, differentiating $n$-times with respect to $a$ on both sides of (8), we obtain

$$
\begin{gathered}
\int_{0}^{\infty} \frac{x^{n} \sin \left(a x+\frac{n \pi}{2}\right)}{\sinh b x} d x \\
=\frac{(-1)^{n} \pi^{n+1}}{b^{n+1}} \cdot \sum_{k=0}^{\infty}(-1)^{k} k^{n} \exp \left(-\frac{a k \pi}{b}\right)
\end{gathered}
$$

Case (B): If $a<0$. Because

$$
\begin{gathered}
\int_{0}^{\infty} \frac{\sin a x}{\sinh b x} d x \\
=\frac{\pi}{2 b} \tanh \frac{a \pi}{2 b} \\
=-\frac{\pi}{2 b} \tanh \left(\frac{-a \pi}{2 b}\right)
\end{gathered}
$$




$$
\begin{array}{r}
=-\frac{\pi}{2 b} \cdot\left[-1+2 \cdot \sum_{k=0}^{\infty}(-1)^{k} \exp \left(\frac{a k \pi}{b}\right)\right] \\
=\frac{\pi}{2 b}-\frac{\pi}{b} \cdot \sum_{k=0}^{\infty}(-1)^{k} \exp \left(\frac{a k \pi}{b}\right)
\end{array}
$$

Also, by differentiation term by term and differentiation with respect to a parameter, differentiating $n$-times with respect to $a$ on both sides of (9), we obtain

$$
\begin{gathered}
\int_{0}^{\infty} \frac{x^{n} \sin \left(a x+\frac{n \pi}{2}\right)}{\sinh b x} d x \\
=-\frac{\pi^{n+1}}{b^{n+1}} \cdot \sum_{k=0}^{\infty}(-1)^{k} k^{n} \exp \left(\frac{a k \pi}{b}\right) \text { q.e.d. }
\end{gathered}
$$

The following is the second major result in this paper, we find the infinite series form of the improper integral (4).

\subsection{Theorem 2}

Let the assumptions be the same as Theorem 1 .

Case (A): If $a>0$. Then the improper integral

$$
\begin{gathered}
\int_{0}^{\infty} \frac{x^{n} \cos \left(a x+\frac{n \pi}{2}\right)}{\cosh b x} d x \\
=\frac{(-1)^{n} \pi^{n+1}}{2^{n} b^{n+1}} \cdot \sum_{k=0}^{\infty}(-1)^{k}(2 k+1)^{n} \exp \left(-\frac{a(2 k+1) \pi}{2 b}\right)
\end{gathered}
$$

Case (B): If $a<0$. Then

$$
\begin{gathered}
\int_{0}^{\infty} \frac{x^{n} \cos \left(a x+\frac{n \pi}{2}\right)}{\cosh b x} d x \\
=\frac{\pi^{n+1}}{2^{n} b^{n+1}} \cdot \sum_{k=0}^{\infty}(-1)^{k}(2 k+1)^{n} \exp \left(\frac{a(2 k+1) \pi}{2 b}\right)
\end{gathered}
$$

\subsubsection{Proof}

Case (A): If $a>0$. Because

$$
\begin{gathered}
\int_{0}^{\infty} \frac{\cos a x}{\cosh b x} d x \\
=\frac{\pi}{2 b} \operatorname{sech} \frac{a \pi}{2 b}(\operatorname{By}(2)) \\
=\frac{\pi}{b} \cdot \frac{\exp \left(\frac{a \pi}{2 b}\right)+\exp \left(-\frac{a \pi}{2 b}\right)}{1} \\
=\frac{\pi}{b} \cdot \exp \left(-\frac{a \pi}{2 b}\right) \cdot \frac{1+\exp \left(-\frac{a \pi}{b}\right)}{\frac{\pi}{b} \cdot \exp \left(-\frac{a \pi}{2 b}\right) \cdot \sum_{k=0}^{\infty}(-1)^{k} \exp \left(-\frac{a k \pi}{b}\right)} \\
\text { geometric series })
\end{gathered}
$$

$$
=\frac{\pi}{b} \cdot \sum_{k=0}^{\infty}(-1)^{k} \exp \left(-\frac{a(2 k+1) \pi}{2 b}\right)
$$

Using differentiation term by term and differentiation with respect to a parameter, differentiating $n$-times with respect to $a$ on both sides of (12), we have

$$
\begin{gathered}
\int_{0}^{\infty} \frac{x^{n} \cos \left(a x+\frac{n \pi}{2}\right)}{\cosh b x} d x \\
=\frac{(-1)^{n} \pi^{n+1}}{2^{n} b^{n+1}} \cdot \sum_{k=0}^{\infty}(-1)^{k}(2 k+1)^{n} \exp \left(-\frac{a(2 k+1) \pi}{2 b}\right)
\end{gathered}
$$

Case (B): If $a<0$. Because

$$
\begin{array}{r}
\int_{0}^{\infty} \frac{\cos a x}{\cosh b x} d x \\
=\frac{\pi}{2 b} \operatorname{sech} \frac{a \pi}{2 b} \\
=\frac{\pi}{2 b}\left(\sec h \frac{-a \pi}{2 b}\right) \\
=\frac{\pi}{b} \cdot \sum_{k=0}^{\infty}(-1)^{k} \exp \left(\frac{a(2 k+1) \pi}{2 b}\right)
\end{array}
$$

Also, by differentiation term by term and differentiation with respect to a parameter, differentiating $n$-times with respect to $a$ on both sides of (13), we obtain

$$
\begin{gathered}
\int_{0}^{\infty} \frac{x^{n} \cos \left(a x+\frac{n \pi}{2}\right)}{\cosh b x} d x \\
=\frac{\pi^{n+1}}{2^{n} b^{n+1}} \cdot \sum_{k=0}^{\infty}(-1)^{k}(2 k+1)^{n} \exp \left(\frac{a(2 k+1) \pi}{2 b}\right) \text { q.e.d. }
\end{gathered}
$$

\section{Examples}

In the following, for the two types of improper integrals in this study, we provide four examples and use Theorems 1, 2 to determine their infinite series forms. On the other hand, we employ Maple to calculate the approximations of these improper integrals and their solutions for verifying our answers.

\subsection{Example 1}

In Theorem 1, let $a=6, b=2, n=8$. By Case (A) of Theorem 1, we obtain the improper integral

$$
\int_{0}^{\infty} \frac{x^{8} \sin 6 x}{\sinh 2 x} d x=\frac{\pi^{9}}{2^{9}} \cdot \sum_{k=0}^{\infty}(-1)^{k} k^{8} \exp (-3 k \pi)
$$

Next, we use Maple to verify the correctness of (14). $>\operatorname{evalf}\left(\operatorname{int}\left(\mathrm{x}^{\wedge} 8 * \sin \left(6^{*} \mathrm{x}\right) / \sinh (2 * \mathrm{x}), \mathrm{x}=0\right.\right.$..infinity), 14);

$$
-0.0046015343323402
$$

$>\operatorname{evalf}\left(\mathrm{Pi}^{\wedge} 9 / 2^{\wedge} 9^{*} \operatorname{sum}\left((-1)^{\wedge} \mathrm{k}^{*} \mathrm{k}^{\wedge} 8^{*} \exp \left(-3 * \mathrm{k}^{*} \mathrm{Pi}\right), \mathrm{k}=0\right.\right.$. 
infinity), 14);

\section{$-0.0046015343323412$}

\subsection{Example 2}

In Theorem 1, taking $a=-4, b=5, n=9$. Using Case (B) of Theorem 1, we can determine the improper integral

$$
\int_{0}^{\infty} \frac{x^{9} \cos 4 x}{\sinh 5 x} d x=-\frac{\pi^{10}}{5^{10}} \cdot \sum_{k=0}^{\infty}(-1)^{k} k^{9} \exp \left(-\frac{4 k \pi}{5}\right)
$$

We also use Maple to verify the correctness of (15). $>$ evalf(int $\left(x^{\wedge} 9 * \cos (4 * x) / \sinh \left(5^{*} x\right), x=0\right.$..infinity $\left.), 14\right)$;

\subsection{4}

$>\operatorname{evalf}\left(-\mathrm{Pi}^{\wedge} 10 / 5^{\wedge} 10^{*} \operatorname{sum}\left((-1)^{\wedge} \mathrm{k}^{*} \mathrm{k}^{\wedge} 9^{*} \exp \left(-4 * \mathrm{k}^{*} \mathrm{Pi} / 5\right), \mathrm{k}=0 . . \mathrm{i}\right.\right.$ nfinity), 14);

\subsection{8}

\subsection{Example 3}

In Theorem 2, let $a=7, b=4, n=12$. By Case (A) of Theorem 2, we can evaluate the improper integral

$$
\begin{gathered}
\int_{0}^{\infty} \frac{x^{12} \cos 7 x}{\cosh 4 x} d x \\
=\frac{\pi^{13}}{2^{12} \cdot 4^{13}} \cdot \sum_{k=0}^{\infty}(-1)^{k}(2 k+1)^{12} \exp \left(-\frac{(14 k+7) \pi}{8}\right)
\end{gathered}
$$

Using Maple to verify the correctness of (16) as follows: $>$ evalf(int $\left(\mathrm{x}^{\wedge} 12 * \cos (7 * \mathrm{x}) / \cosh (4 * \mathrm{x}), \mathrm{x}=0\right.$...infinity), 14);

\subsection{4}

$>\operatorname{evalf}\left(\mathrm{Pi}^{\wedge} 13 /\left(2^{\wedge} 12^{*} 4^{\wedge} 13\right) * \operatorname{sum}\left((-1)^{\wedge} \mathrm{k}^{*}\left(2^{*} \mathrm{k}+1\right)^{\wedge} 12^{*} \exp (-(\right.\right.$ $14 * \mathrm{k}+7) * \mathrm{Pi} / 8), \mathrm{k}=0$..infinity), 14$)$;

\subsection{9}

\subsection{Example 4}

In Theorem 2, let $a=-6, b=8, n=15$. Using Case (B) of Theorem 2, we obtain the improper integral

$$
\begin{gathered}
\int_{0}^{\infty} \frac{x^{15} \sin 6 x}{\cosh 8 x} d x \\
=-\frac{\pi^{16}}{2^{15} \cdot 8^{16}} \cdot \sum_{k=0}^{\infty}(-1)^{k}(2 k+1)^{15} \exp \left(-\frac{(6 k+3) \pi}{8}\right)
\end{gathered}
$$

We also use Maple to verify the correctness of (17). $>\operatorname{evalf}\left(\operatorname{int}\left(\mathrm{x}^{\wedge} 15^{*} \sin \left(6^{*} \mathrm{x}\right) / \cosh \left(8^{*} \mathrm{x}\right), \mathrm{x}=0\right.\right.$...infinity $\left.), 18\right)$;

$$
-0.000200107507162211911
$$

$>\operatorname{evalf}\left(-\mathrm{Pi}^{\wedge} 16 /\left(2^{\wedge} 15^{*} 8^{\wedge} 16\right) * \operatorname{sum}\left((-1)^{\wedge} \mathrm{k}^{*}\left(2^{*} \mathrm{k}+1\right)^{\wedge} 15^{*} \exp (-(\right.\right.$
$6 * \mathrm{k}+3) * \mathrm{Pi} / 8), \mathrm{k}=0$...infinity), 18);

$$
-0.000200107507162211670
$$

\section{Conclusions}

As mentioned, the geometric series, the differentiation term by term, and the differentiation with respect to a parameter play significant roles in the theoretical inferences of this study. In fact, the applications of these theorems are extensive, and can be used to easily solve many difficult problems; we endeavor to conduct further studies on related applications. On the other hand, Maple also plays a vital assistive role in problem-solving. In the future, we will extend the research topic to other calculus and engineering mathematics problems and solve these problems by using Maple. These results will be used as teaching materials for Maple on education and research to enhance the connotations of calculus and engineering mathematics.

\section{REFERENCES}

[1] M. L. Abell and J. P. Braselton, Maple by Example, 3rd ed., New York: Elsevier Academic Press, 2005.

[2] J. S. Robertson, Engineering Mathematics with Maple, New York: McGraw-Hill, 1996.

[3] F. Garvan, The Maple Book, London: Chapman \& Hall/CRC, 2001.

[4] R. J. Stroeker and J. F. Kaashoek, Discovering Mathematics with Maple : An Interactive Exploration for Mathematicians, Engineers and Econometricians, Basel: Birkhauser Verlag, 1999.

[5] C. T. J. Dodson and E. A. Gonzalez, Experiments in Mathematics Using Maple, New York: Springer-Verlag, 1995.

[6] D. Richards, Advanced Mathematical Methods with Maple, New York: Cambridge University Press, 2002.

[7] C. Tocci and S. G. Adams, Applied Maple for Engineers and Scientists, Boston: Artech House, 1996.

[8] D. Zwillinger (Ed.), CRC Standard Mathematical Tables and Formulae, 31st ed., Boca Raton: Chapman \& Hall/CRC, p455, 2003.

[9] A. A. Adams, H. Gottliebsen, S. A. Linton, and U. Martin, Automated theorem proving in support of computer algebra: symbolic definite integration as a case study, Proceedings of 1999 International Symposium on Symbolic and Algebraic Computation, pp. 253-260, Vancouver, Canada, 1999.

[10] M. A. Nyblom, On the evaluation of a definite integral involving nested square root functions, Rocky Mountain Journal of Mathematics, Vol. 37, No. 4, pp. 1301-1304, 2007.

[11] C. Oster, Limit of a definite integral, SIAM Review, Vol. 33, No. 1, pp. 115-116, 1991. 
[12] C.-H. Yu, Power series forms of four types of improper integrals, Chia Nan Annual Bulletin, Vol. 38, pp. 241-248, 2012.

[13] C.-H. Yu, The improper integral problems related to hyperbolic functions, Journal of Tzu Hui, Vol. 8, pp. 192-203, 2012.

[14] C.-H. Yu, Generalization of some improper integral formulas, Cheng Shiu Journal of General Education, Vol. 10, pp. 25-42, 2013.

[15] C.-H. Yu, The series expressions of some integrals, Yu Da Academic Journal, Vol. 32, pp.93-103, 2012.

[16] C.-H. Yu, Application of Maple: taking two types of improper integral problems as examples, Proceedings of 2013 Information Technology and Management Symposium, B9-B14, Taiwan, 2013.

[17] C.-H. Yu, Application of Maple on some type of improper integral problem, Proceedings of 2012 Workshop on Consumer Electronics, P0081, Taiwan, 2012.

[18] C. -H. Yu, Using Maple to study two types of integrals, International Journal of Research in Computer Applications and Robotics, Vol. 1, Issue. 4, pp. 14-22, 2013.

[19] C.-H. Yu, Application of Maple on evaluation of definite integrals, Applied Mechanics and Materials, in press, 2013.

[20] C. -H. Yu, A study on integral problems by using Maple, International Journal of Advanced Research in Computer Science and Software Engineering, Vol. 3, Issue. 7, pp. 41-46, 2013.

[21] C. -H. Yu, Evaluating some integrals with Maple, International Journal of Computer Science and Mobile Computing, Vol. 2, Issue. 7, pp. 66-71, 2013.

[22] C.-H. Yu, Application of Maple on some integral problems,
Proceedings of the International Conference on Safety \& Security Management and Engineering Technology 2012, pp. 290-294, 2012.

[23] C.-H. Yu, Application of Maple: taking two special integral problems as examples, Proceedings of the 8th International Conference on Knowledge Community, pp.803-811, 2012.

[24] C.-H. Yu, Application of Maple on evaluating the closed forms of two types of integrals, Proceedings of the 17th Mobile Computing Workshop, ID16, 2012.

[25] C.-H. Yu, Application of Maple on the integral problem of some type of rational functions, Proceedings of the Annual Meeting and Academic Conference for Association of IE, D357-D362, 2012.

[26] C. -H. Yu, Solving some integrals with Maple, International Journal of Research in Aeronautical and Mechanical Engineering, Vol. 1, Issue. 3, pp. 29-35, 2013.

[27] C. -H. Yu, Using Maple to study the integrals of trigonometric functions, Proceedings of the 6th IEEE/International Conference on Advanced Infocomm Technology, No. 00294, 2013.

[28] C. -H. Yu, A study of the integrals of trigonometric functions with Maple, Proceedings of the Institute of Industrial Engineers Asian Conference 2013, Springer, Vol. 1, pp. 603-610, 2013.

[29] C.-H. Yu, Application of Maple on some type of integral problem, Proceedings of the Ubiquitous-Home Conference 2012, pp.206-210, 2012.

[30] T. M. Apostol, Mathematical Analysis, 2nd ed., Boston: Addison-Wesley, p230, 1975.

[31] L. Flatto, Advanced Calculus, Baltimore: The Williams \& Wilkins, p405, 1976. 